\title{
Clinical characteristics and long-term visual outcome of severe phenotypes of chronic central serous chorioretinopathy
}

This article was published in the following Dove Press journal:

Clinical Ophthalmology

\author{
Danial Mohabati ${ }^{1,2}$ \\ Thomas J van Rijssen' \\ Elon HC van Dijk' \\ Gregorius PM Luyten' \\ Tom O Missotten ${ }^{3}$ \\ Carel B Hoyng ${ }^{4}$ \\ Suzanne Yzer ${ }^{3}$ \\ Camiel JF Boon ${ }^{1,5}$ \\ 'Department of Ophthalmology, \\ Leiden University Medical Center, \\ Leiden, the Netherlands; ${ }^{2}$ Department \\ of Ophthalmology, Rotterdam \\ Ophthalmic Institute, Rotterdam, \\ the Netherlands; ${ }^{3}$ Department of \\ Ophthalmology, The Rotterdam Eye \\ Hospital, Rotterdam, the Netherlands; \\ ${ }^{4}$ Department of Ophthalmology, \\ Donders Institute for Brain, \\ Cognition and Behaviour, Radboud \\ University Medical Center, Nijmegen, \\ the Netherlands; ${ }^{5}$ Department of \\ Ophthalmology, Academic Medical \\ Center, University of Amsterdam, \\ Amsterdam, the Netherlands
}

Correspondence: Camiel JF Boon Department of Ophthalmology, Leiden University Medical Center, Albinusdreef 2, 2333 ZA Leiden, the Netherlands

$\mathrm{Tel}+3|7| 5265936$

Fax +3I 7I 5248222

Email c.j.f.boon@lumc.nl
Purpose: The aim of this study was to investigate disease onset and disease progression in patients with severe chronic central serous chorioretinopathy (cCSC).

Patients and methods: The medical records of $143 \mathrm{cCSC}$ patients (199 eyes) were reviewed. All cases had visual complaints for $>6$ months and showed signs of a severe disease phenotype on optical coherence tomography (OCT) and fluorescein angiography (FA). Clinical presentation at onset was evaluated, together with disease progression on multimodal imaging and final treatment outcome.

Results: Twenty-eight cases (14\%) had a documented history of an acute episode of CSC, whereas 145 cases $(73 \%)$ showed pre-existing features of chronicity already at first presentation. The first clinical presentation could not be evaluated in 13\% of cases. Best-corrected visual acuity (BCVA) was $70 \pm 18$ Early Treatment of Diabetic Retinopathy Study (ETDRS) letters at onset and $70 \pm 22$ ETDRS letters at final visit ( $p=0.770)$. Among all studied cases, 173 eyes $(87 \%)$ were treated, which resulted in complete resolution of subretinal fluid (SRF) in $76 \%$ of eyes at final visit. In eyes with fluorescein angiographic follow-up, the area of diffuse atrophic retinal pigment epithelium (RPE) abnormalities (diffuse atrophic RPE alterations [DARA]) had increased significantly in 43 eyes $(68 \%)$ at final visit.

Conclusion: CSC encompasses a clinical spectrum that includes a range of severe phenotypes, in which retinal abnormalities tend to be progressive. Nevertheless, the long-term visual acuity may remain fairly stable with treatment. Few patients with severe chronic CSC have a history of acute CSC, which could indicate that there may be pathogenetic differences between these 2 CSC variants.

Keywords: chronic central serous chorioretinopathy, long-term outcome, severity, treatment, photodynamic therapy, posterior cystoid retinal degeneration

\section{Introduction}

Central serous chorioretinopathy (CSC) is characterized by a serous detachment of the neurosensory retina. ${ }^{1}$ Although the precise pathophysiology of CSC is unclear, a congested and hyperpermeable choriocapillaris and thickened choroid (pachychoroid) may be the primary source of serous fluid leakage. ${ }^{2}$ Additionally, it is hypothesized that due to secondary retinal pigment epithelium (RPE) alterations, the outer blood-retinal barrier is dysfunctional, which subsequently leads to fluid passage into the subretinal space. ${ }^{3}$ A prolonged presence of subretinal fluid (SRF) can lead to irreversible photoreceptor damage and dysfunction, ${ }^{4}$ resulting in visual complaints such as blurry vision, metamorphopsia, dyschromatopsia, micropsia, macropsia, and disturbed contrast vision. Men in the professional age range are affected most frequently. ${ }^{5}$ Thus far, the most important identified risk factors include the use of corticosteroids, endogenous hypercortisolism, 
mental stress, and pregnancy. ${ }^{6}$ Genetic risk factors have also been shown to be associated with $\mathrm{CSC}^{7-9}$

Although there is no generally accepted classification of CSC, 2 basic forms of CSC are often distinguished: acute and chronic CSC (cCSC). ${ }^{4,10}$ The acute phenotype of CSC is typically defined as an acute episode of central vision loss due to SRF leakage, with a single "hot spot" of leakage through the RPE seen on fluorescein angiography (FA), without any atrophic RPE abnormalities. In this definition, acute CSC is generally self-limiting, with spontaneous resolution within 3-6 months after onset and a favorable visual prognosis. ${ }^{10}$ In contrast, patients with cCSC do not tend to have a spontaneous resolution of SRF, have a more unfavorable visual prognosis, ${ }^{11}$ and a variable clinical manifestation. ${ }^{10}$ Some patients with cCSC may present with relatively limited atrophic RPE alterations, focal RPE detachments, and focal leakage.

There are other cases that show more severe forms of cCSC. They show widespread areas of RPE atrophy, larger and more numerous RPE detachments, diffuse areas of leakage through the RPE, and posterior cystoid retinal degeneration (PCRD), and in rare cases even inferior bullous retinal detachments are present. ${ }^{12-14}$ In the past, the term diffuse retinal pigment epitheliopathy has also been used to distinguish this severe disease course from more acute forms of CSC. ${ }^{15}$ Little is known about the clinical characteristics, the long-term outcome, and response to treatment in these severe cases of cCSC. Also, it is unclear whether severe cases of cCSC are generally preceded by previous episodes of typical acute CSC. As a consequence, there is currently an ongoing debate on whether acute CSC and (severe) cCSC are either part of a clinical and pathophysiological continuous spectrum, or if these phenotypes are more distinct with regard to their background, presentation, and treatment outcome.

In the present study, we describe a large group of cCSC cases that show phenotypic signs of severity. The aim of the study was to describe the range of clinical findings on multimodal imaging in these presumably severe cCSC cases and to evaluate the long-term visual outcome and treatment response. Finally, we assess whether or not these severe cCSC cases were preceded by typical acute CSC.

\section{Patients and methods}

\section{Patient selection}

In this retrospective multicenter study, patients were included from 3 Dutch tertiary referral centers: the Department of Ophthalmology of Leiden University Medical Center (Leiden, the Netherlands), the Rotterdam Eye Hospital (Rotterdam, the Netherlands), and the Department of Ophthalmology of Radboud University Medical Center (Nijmegen, the Netherlands). Study patients were selected from a cohort of 1,387 subjects who were diagnosed with acute CSC and cCSC between 2005 and 2016. For the purpose of this study, we only included cCSC. Chronicity was defined as persistence of visual complaints for $>6$ months, in the presence of anatomical abnormalities compatible with typical chronic CSC ${ }^{8}$ such as multifocal diffuse RPE alterations and chronic SRF leakage based on multimodal imaging that included optical coherence tomography (OCT), FA, and/or indocyanine green angiography (ICGA). In addition, a cCSC case was considered severe when at least 1 of the following abnormalities within the anatomical macular area inside the largest temporal vascular arcades (Figure 1) was present: 1) cumulative areas of $>5$ optic disc diameters (DD) of diffuse atrophic RPE alterations (DARA) as visualized on mid-phase FA; 2) at least 2 "hot spots" of leakage separated by at least 1 DD of non-hyperfluorescent healthy-appearing retina on mid-phase FA (multifocal "hot spots"); 3) an area of diffuse fluorescein leakage with a surface of $>1$ DD on mid-phase FA, without an evident leaking focus (diffuse leakage); and 4) presence of PCRD assessed on OCT. ${ }^{12}$ All included cases had to have at least 1 of these severe criteria, but could have up to all 4 criteria. Subjects were excluded when there was a suspicion of a (secondary) choroidal neovascularization and/or polypoidal choroidal vasculopathy or in the case of evidence of other underlying retinal diseases such as age-related macular degeneration (with presence of drusen in both eyes), multifocal choroiditis, retinal vascular occlusions, pseudoxanthoma elasticum, amblyopia, or high myopia ( $>6$ diopters).

\section{Clinical outcome measures}

Retrospective data were collected on the patient's first presentation, based on available multimodal imaging (at least FA and OCT). This information was used to distinguish between an acute and a chronic first manifestation of CSC. RPE detachments outside the area of SRF leakage, evidence of RPE atrophy (visible as window defects on FA), and/or multifocal hot spots or diffuse fluorescein leakage were seen as signs of chronicity. In contrast, a presentation with one well-defined pinpoint leakage on FA, lack of any RPE alterations, and a sudden neurosensory detachment followed by a spontaneous recovery in $<6$ months were considered to represent a typical acute CSC episode. In case no images at first presentation were available, the clinical description by the treating ophthalmologist was used to distinguish between an acute and a chronic first presentation. Furthermore, information was collected on all treatment strategies and treatment response, the progression of 

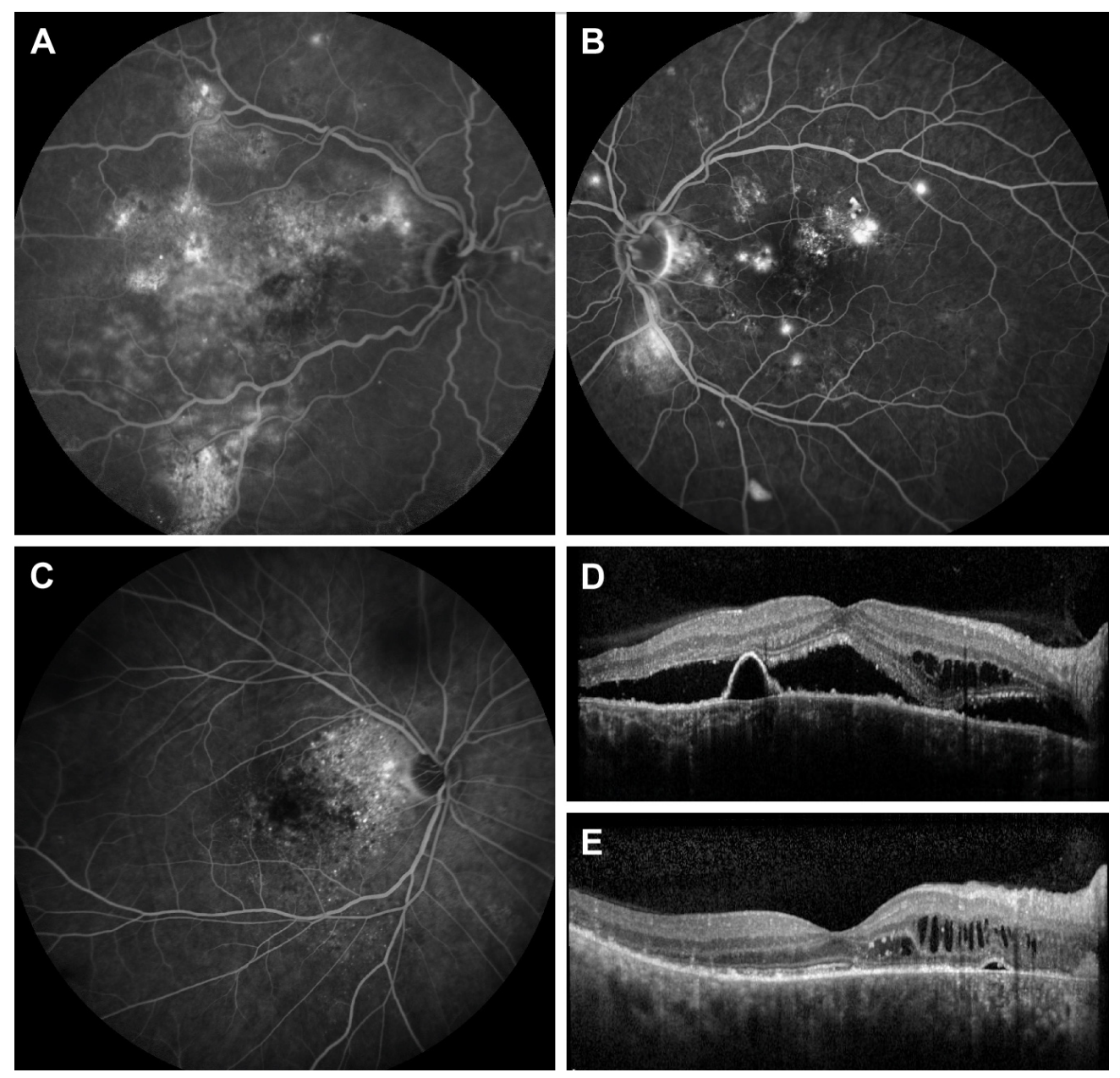

Figure I Illustration of the 4 criteria of severity on FA and OCT.

Notes: The 4 criteria are as follows: I) cumulative areas of $>5$ DD of diffuse atrophic retinal pigment epithelium alterations as visualized on mid-phase FA (A). 2) Multiple (at least 2) "hot spots" of leakage separated by at least I DD of non-hyperfluorescent healthy-appearing retina on mid-phase FA (B). 3) An area of diffuse fluorescein leakage with a surface of $>$ I DD on mid-phase FA, without an evident leaking focus (C). 4) Presence of posterior cystoid retinal degeneration on OCT (D and E).

Abbreviations: DD, optic disc diameters; FA, fluorescein angiography; OCT, optical coherence tomography.

atrophic retinal abnormalities, and visual acuity at presentation (baseline), and at last available follow-up visit (final follow-up). Information on the use of steroid-containing medication and endogenous hypercortisolism was collected. The extent of atrophic retinal abnormalities as DARA surface was quantified on FA images by using the available caliper measurement tools on FA equipment and expressed in number of DD. The location of DARA was identified and categorized as follows: 1) including the fovea, 2) within $1 \mathrm{DD}$ of the fovea, but sparing the fovea, and 3) $>1$ DD outside the fovea. The location of hot spots of leakage on FA was described as either inside or outside the largest temporal retinal vascular arcade. Central foveal thickness (CFT) was measured manually in patients in whom a spectral-domain OCT was available. CFT was defined as the distance from the inner border of the internal limiting membrane (ILM) to the inner border of the ellipsoid zone (EZ) to minimize measurement errors due to the presence of subretinal accumulation of debris and SRF in active cCSC. CFT was evaluated at the moment of diagnosis of severe cCSC and compared to the final visit.

\section{Clinical examinations}

All patients underwent a range of ophthalmological and multimodal imaging examinations at the moment of diagnosis and during follow-up. This included best-corrected visual acuity (BCVA) (measured with Snellen charts, which was converted to Early Treatment of Diabetic Retinopathy Study (ETDRS) letters for statistical comparisons), ${ }^{16}$ slit lamp examination, and/or color fundus photography (either Topcon Corp., Tokyo, Japan or Carl Zeiss Meditec AG, Jena, Germany), either timedomain OCT (either Cirrus HD-OCT; Carl Zeiss Meditec AG, or OCT-HS100; Canon Inc, Tokyo, Japan) or spectral-domain OCT (Spectralis HRA + OCT; Heidelberg Engineering, Heidelberg, Germany), FA (Heidelberg Engineering, Topcon 
Corp., or Carl Zeiss Meditec AG), and ICGA (Spectralis HRA + OCT; either Topcon Corp or Carl Zeiss Meditec AG).

\section{Treatment}

In this retrospective study, the decision for treatment, retreatment, and treatment modality was based on the preference of the treating ophthalmologist. Treatment was applied when there was persistent SRF affecting vision. Photodynamic therapy (PDT) was performed with different settings such as half-dose (3 mg/m² verteporfin [Visudyne ${ }^{\circledR}$; Novartis international AG, Basel, Switzerland]), half-time (treatment duration of 42 seconds), half-fluency $\left(25 \mathrm{~J} / \mathrm{cm}^{2}\right)$, or the original settings $\left(6 \mathrm{mg} / \mathrm{m}^{2}\right.$ verteporfin, 83 seconds, and $\left.50 \mathrm{~J} / \mathrm{cm}^{2}\right)$ as described for neovascular age-related macular degeneration. Other reviewed treatments included subthreshold micropulse diode laser (SML) and conventional thermal laser.

\section{Statistical analysis}

Statistical analysis was performed using IBM SPSS software for Windows, version 23 (IBM Corporation, Armonk, NY, USA). Either a paired samples $t$-test or a one-way analysis of variance (ANOVA) test was used for comparing mean values in continuous numerical data. Categorical data were analyzed using either a Chi-square test or a McNemar Chi-square test. Multivariate regression analyses were performed using a forward stepwise linear regression, where the final BCVA outcome was used as a dependent variable and multiple-associated clinical findings (as will be described later on) as explanatory variables. The best linear model for grading of discrepancy decrease was calculated. A $p$-value of $<0.05$ was considered significant in all performed tests.

\section{Ethics approval}

All procedures performed in this study involving human participants were in accordance with the ethical standards of the institutional and/or national research committee and with the 1964 Declaration of Helsinki and its later amendments or comparable ethical standards. The local institutional review boards in all participating centers ('Medisch Ethische Toetsingscommissie' (METC) in Leiden University Medical Center, The METC in the Radboud University Medical Center, and the 'Wetenschapscommissie' in the Rotterdam Eye Hospital) did not require written consent from the participants for reviewing their medical records, as all data were anonymized upon collection.

\section{Results}

After a review of the medical charts and clinical characteristics on multimodal imaging, a total of 143 patients (199 eyes,
Table I Patient demographics

\begin{tabular}{ll}
\hline Characteristics & Cases \\
\hline Patients (eyes) & $143(199)$ \\
Male gender, $\mathrm{n}(\%)$ & $\mathrm{I} 2 \mathrm{I}(87)$ \\
Caucasian ethnicity, $\mathrm{n}(\%)$ & $\mathrm{I} 19(83)$ \\
Mean age at diagnosis of CSC (range) & $46(26-78)$ \\
Mean available follow-up in years (range) & $7.5(0.3-30.6)$ \\
Patients with recent ${ }^{\mathrm{b}}$ use of steroids, $\mathrm{n}(\%)$ & $25^{\mathrm{c}}(18)$ \\
BCVA at first presentation (ETDRS letters) & $70 \pm 18$ \\
\hline
\end{tabular}

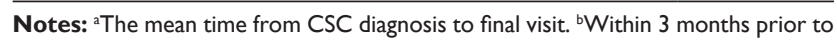
diagnosis. 'One patient was diagnosed with Cushing syndrome. Data presented as mean $\pm S D$ unless otherwise indicated.

Abbreviations: BCVA, best-corrected visual acuity; CSC, central serous chorioretinopathy; ETDRS, Early Treatment of Diabetic Retinopathy Study.

$87 \%$ males, $83 \%$ Caucasians) with cCSC could be included who met our definition of severity in at least 1 eye. Mean age at diagnosis of CSC was 46 years, and mean follow-up duration was 7.5 years (Table 1). At final follow-up, 56 patients (39\%) had a severe cCSC phenotype in both eyes. Severity manifested on average after 5.6 months (0-94 months) in the second eye in this subgroup. After reviewing data on the very first presentation of CSC, 28 eyes (14\%) started with an acute episode with limited or no RPE damage and spontaneous resolution of SRF. However, most of the included subjects (145 eyes, $73 \%$ ) already showed a chronic phenotype at first presentation, with $\geq 1$ significant multifocal areas of atrophic RPE alterations and/or multifocal areas of leakage. In 26 eyes (13\%), insufficient information from the primary disease manifestation was available.

\section{Characteristics on multimodal imaging}

In 133 eyes (67\%), > 1 criterion of severity was present at some point during the follow-up. The distribution of these criteria of severity is summarized in Table 2. A gravitational tract was observed in 84 eyes (42\%) on FA. Fifteen eyes ( $8 \%$ ) had a secondary macular epiretinal membrane without evidence of diseases other than CSC explaining the membrane. Fundus photography, OCT, and FA revealed choroidal folds in 3 eyes (2\%), without a hyperopic refractive error. Hard exudates were observed in 6 eyes (3\%) without any sign of retinal (neo) vascular abnormalities or polypoidal choroidal vasculopathy on FA and ICGA. In 1 patient ( 1 eye, $0.5 \%$ ), a bullous inferior retinal detachment secondary to $\mathrm{cCSC}$ was present (Figure 2). ICGA images were available in 134 of the studied eyes (67\%). When comparing the cumulative area of hyperfluorescent RPE alterations and leakage on FA with the area(s) of hyperfluorescent choroidal hyperpermeability on ICGA in these 134 eyes, the cumulative area of abnormalities was larger on ICGA as compared to FA in 79 eyes $(59 \%, 79 / 134)$, whereas in 50 eyes $(37 \%, 50 / 134)$ these areas were equal in size. A smaller 
Table 2 Distribution of each criterion of severity among the severe cases of chronic central serous chorioretinopathy

\begin{tabular}{ll}
\hline Criteria & Frequency and specifications \\
\hline Eyes with DARA, $\mathrm{n}(\%)$ & $145(73)$ \\
Mean DARA surface (DD) & $8.6 \pm 4$ \\
Location of DARA, $\mathrm{n}(\%)$ & $75(52)$ covering the fovea \\
& $47(32)$ within I DD of the fovea \\
& $23(16)$ outside I DD of the fovea \\
Eyes with PCRD, $\mathrm{n}(\%)$ & $69(35)$ \\
Eyes with multiple hot spots, $\mathrm{n}(\%)$ & $115(58)$ \\
Mean number of hot spots (range) & $3(2-7)$ \\
Location of hot spots, $\mathrm{n}(\%)$ & $88(75)$ inside the temporal vascular \\
& arcade \\
& $3(3)$ outside the temporal vascular \\
arcade \\
Eyes with diffuse leakage, $\mathrm{n}(\%)$ & $26(22)$ both inside and outside \\
\hline
\end{tabular}

Notes: aDARA surface overlaps with the area as wide as I DD around the fovea, but does not cover the foveal depression. Data presented as mean \pm SD unless otherwise indicated.

Abbreviations: DARA, diffuse atrophic retinal pigment epithelium (RPE) alterations; $\mathrm{DD}$, optic disc diameters; PCRD, posterior cystoid retinal degeneration.

cumulative area of hyperfluorescent abnormalities on ICGA as compared to FA was present in only 5 eyes $(4 \%, 5 / 134)$.

\section{BCVA}

Mean BCVA in this cohort was $70( \pm 18)$ ETDRS letters at first presentation (baseline BCVA). BCVA at the moment of diagnosis of severe cCSC, on average 4 years after first presentation, was $68( \pm 19)$ ETDRS letters. The final mean BCVA, on average 5.2 years (range: $0.1-25$ years, median $=2.8$ years) after first presentation, was $70( \pm 22)$ ETDRS letters, which was not statistically different in comparison with baseline (paired samples $t$-test, $p=0.770$ ) and with the moment of diagnosis of severe CSC ( $p=0.061)$ (Table 3$)$. Among clinical characteristics (Table 4), final BCVA correlated most significantly with the location of DARA in the macula $(r=0.4$, Pearson correlation, $p<0.001$ ) (Table 4). Mean final BCVA in eyes showing DARA with foveal involvement was significantly worse ( $63 \pm 22$ ETDRS letters) compared to both eyes with DARA located within 1 DD of the fovea but excluding the fovea ( $74 \pm 19$ ETDRS letters, one-way ANOVA, $p=0.003$ ) and eyes with DARA outside 1 DD of the fovea $(78 \pm 18$ ETDRS letters, $p<0.001)$. Mean final BCVA did not significantly differ among eyes with DARA located within 1 DD of the fovea - but excluding the fovea compared to eyes with DARA $>1$ DD away from the fovea $(p=0.582)$. In 9 eyes $(5 \%)$, a BCVA drop $>2$ ETDRS lines was observed after PDT with reduced settings. All of these eyes showed central DARA with foveal involvement. BCVA in 3 out of these 9 eyes $(2 \%, 3 / 199)$ never recovered after PDT until final follow-up. In the first patient, BCVA had decreased from 83 to 70 ETDRS letters, in the second patient from 77 to 65 ETDRS letters, and in the third patient from 70 to 59 ETDRS letters. In 63 eyes (32\%), evolution of DARA could be assessed on a second available FA, which was obtained on average 8 years (range: 1-27 years, median: 7 years) after the first FA images. In 43 of these eyes $(68 \%, 43 / 63)$, the mean surface of DARA had increased on follow-up, from 6.5 DD to $10.3 \mathrm{DD}(p<0.001)$. In 20 eyes $(32 \%, 20 / 63)$, DARA did not change as compared to the previous FA. A multiple linear regression model was computed to predict the final BCVA outcome based on the following parameters: baseline (before severity) BCVA, total area of DARA at first severe presentation, location of DARA, and presence of PCRD, which all correlated independently with final BCVA outcome. A significant regression equation was found $(F(4,167)=36.967, p<0.001)$ with an $R^{2}$ of 0.470 and an adjusted $R^{2}$ of 0.457 . According to this model, baseline BCVA, total area of DARA, location of DARA, and presence of PCRD explain up to $47 \%$ of the observed variance in final BCVA outcome in the present study.

\section{Treatment and disease recurrence}

In this cohort of severe cCSCs, 173 eyes (87\%) were treated at least once (mean: 2.4 treatments, range: 1-20 treatments) from the diagnosis of CSC until final follow-up (Table 5). A majority of these eyes $(83 \%, 143 / 173)$ were treated after the onset of severe disease. Eighty-one of these eyes $(47 \%$, $81 / 173$ ) were treated only once until the final visit. Of these, 41 eyes $(51 \%, 41 / 81)$ never experienced a recurrence after this single treatment. A complete resolution of SRF at final follow-up, on average $3.6 \pm 4$ years after disease onset, was seen in 72 eyes $(89 \%, 72 / 81)$ in this subgroup. Treatment modality and the number of treatments varied widely among the severe cCSC cases (Table 5). Treatment efficacy, defined as a complete resolution of SRF and PCRD, was also highly variable among different treatment modalities (Table 5). In 173 of the 199 eyes (87\%), there was SRF accumulation on OCT at the moment of first severe cCSC presentation. This presence of SRF was significantly less frequent at final visit (47 eyes [24\%], McNemar test, $p<0.001$ ). A comparable significant reduction from $35 \%$ (69 eyes) at severe presentation to $18 \%$ (35 eyes) at final visit was observed in the incidence of PCRD (Table 3). Throughout the follow-up period, 119 eyes $(60 \%)$ had multiple SRF recurrences (mean: 1.2 recurrences, range: $1-9$ recurrences), whereas in 59 eyes $(30 \%)$ no recurrence occurred. In 21 eyes $(10 \%)$, there was insufficient information on disease recurrence. 

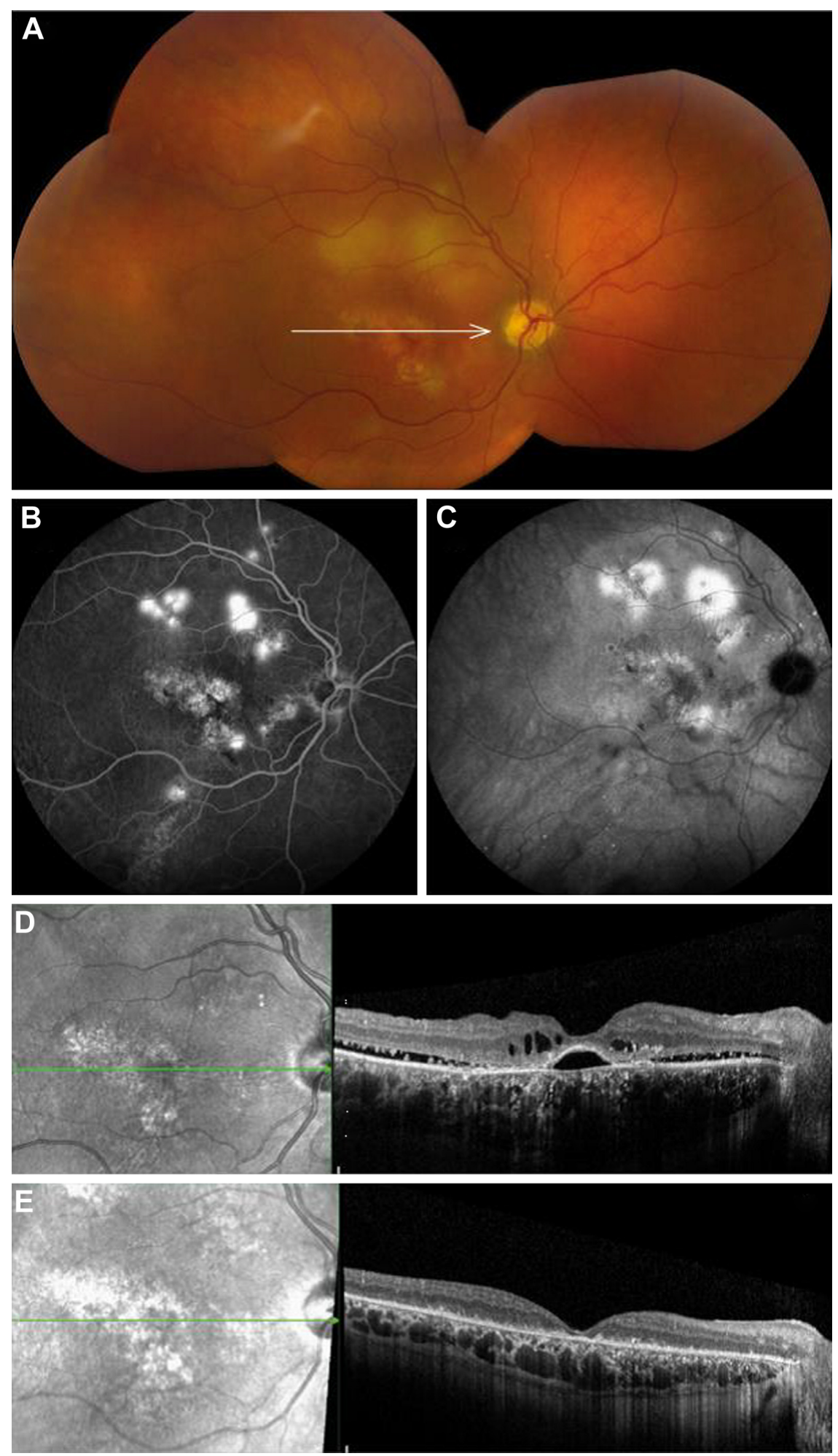

Figure 2 Clinical features on multimodal imaging of the right eye of a 44-year-old male patient with severe chronic central serous chorioretinopathy and a bullous inferior retinal detachment.

Notes: Color fundus photograph showed extensive retinal abnormalities in the macula, with multifocal areas of whitish fibrinous subretinal material (A). The white arrow shows the scanning plane which is depicted on the SD-OCT scans ( $\mathbf{D}$ and $\mathbf{E})$. FA imaging (B) revealed multiple foci of leakage and widespread RPE alterations. The areas of hyperfluorescence on mid-phase ICGA (C) depicted diffuse choroidal hyperpermeability which is larger as compared to the abnormalities on FA. An SD-OCT scan (D) at first presentation and prior to treatment revealed a subretinal SRF accumulation, a subfoveal RPE detachment, and PCRD in the outer nuclear layer of the retina. At $\sim 4$ months after half-dose PDT using a large spot size of I,200 $\mu \mathrm{m}$ centered on the hyperfluorescent zones on ICGA, both SRF and intraretinal fluid on OCT had resolved completely (E). The choroid before treatment was markedly thickened (D). This choroidal thickness reduced after PDT but showed large cavities in the deep choroidal (Haller) layers, with limited or no RPE damage and spontaneous resolution of SRF layers (E).

Abbreviations: FA, fluorescein angiography; ICGA, indocyanine green angiography; PCRD, posterior cystoid retinal degeneration; PDT, photodynamic therapy; RPE, retinal pigment epithelium; SD-OCT, spectral-domain optical coherence tomography; SRF, subretinal fluid. 
Table 3 Characteristics at first presentation and disease progression in severe cases of chronic central serous chorioretinopathy

\begin{tabular}{|c|c|c|c|}
\hline Characteristics & $\begin{array}{l}\text { Severe manifestation } \\
\text { of CSC }\end{array}$ & $\begin{array}{l}\text { Final follow-up } \\
\text { visit }\end{array}$ & $p$-value \\
\hline BCVA (ETDRS letters) & $68 \pm 19$ & $70 \pm 22$ & 0.061 \\
\hline Eyes with SRF, n (\%) & $173(87)$ & $47(24)$ & $<0.001$ \\
\hline Eyes with PCRD, n (\%) & $69(35)$ & $35(18)$ & $<0.001$ \\
\hline $\mathrm{CFT}^{\mathrm{a}}(\mu \mathrm{m})$ & $105 \pm 31$ & $106 \pm 30$ & 0.930 \\
\hline DARA surface ${ }^{\mathrm{b}}(\mathrm{DD})$ & $6.3 \pm 3$ & $9.0 \pm 5$ & $<0.001$ \\
\hline Mean number of treatments ${ }^{c}$ (range) & $\mathrm{n} / \mathrm{a}$ & $2.5(I-20)$ & $\mathrm{n} / \mathrm{a}$ \\
\hline Mean number of recurrences ${ }^{d}$ (range) & $\mathrm{n} / \mathrm{a}$ & I.2 (0-9) & $\mathrm{n} / \mathrm{a}$ \\
\hline
\end{tabular}

Notes: aCFT could be measured manually in 63 eyes (32\%) with a spectral-domain optical coherence tomography. bln 68 cases (34\%), a follow-up FA (at least I year later) was available to be compared with baseline FA. 'Twenty-six cases (I3\%) were never treated and were not included in this analysis. ${ }^{\mathrm{d}}$ Twenty-one cases (II\%) never showed a complete resolution of SRF, and were not included in this analysis. Data presented as mean \pm SD unless otherwise indicated. Bold font indicates data were considered statistically significant. Abbreviations: BCVA, best-corrected visual acuity; CSC, central serous chorioretinopathy; CFT, central foveal thickness; DARA, diffuse atrophic retinal pigment epithelium (RPE) alterations; DD, optic disc diameters; ETDRS, Early Treatment of Diabetic Retinopathy Study; FA, fluorescein angiography; n/a, not applicable; PCRD, posterior cystoid retinal degeneration; SRF, subretinal fluid.

\section{Discussion}

In this retrospective multicenter study, we assessed the clinical characteristics in a large series of eyes with a severe variant of cCSC, after suggesting a definition for severity. A majority of the studied eyes (73\%) manifested pre-existing signs of chronicity at first disease presentation, where only $14 \%$ started with a documented acute CSC episode. In contrast to a high percentage of SRF resolution, final visual

Table 4 Pearson correlation coefficients between patient characteristics and final visual outcome

\begin{tabular}{|c|c|c|c|}
\hline \multirow[t]{2}{*}{ Characteristics } & \multicolumn{3}{|c|}{$\begin{array}{l}\text { Correlation with BCVA } \\
\text { outcome }\end{array}$} \\
\hline & $\boldsymbol{r}$ & $\begin{array}{l}\text { Evaluated } \\
\text { cases }\end{array}$ & $\begin{array}{l}\text { Multivariate } \\
\text { p-value }\end{array}$ \\
\hline Male gender & 0.161 & 197 & 0.024 \\
\hline Age at disease onset & -0.086 & 197 & 0.225 \\
\hline Caucasian ethnicity & -0.045 & 197 & 0.526 \\
\hline Recent steroid use & 0.042 & 197 & 0.551 \\
\hline Disease duration & -0.105 & 197 & 0.143 \\
\hline Baseline BCVA & 0.543 & 197 & $<0.001$ \\
\hline Presence of PCRD & -0.297 & 195 & $<0.001$ \\
\hline Presence of multifocal hot spots & 0.023 & 195 & 0.023 \\
\hline Mean number of hot spots & -0.036 & 152 & 0.656 \\
\hline Location of multifocal hot spots & -0.036 & 154 & 0.657 \\
\hline Presence of diffuse leakage & -0.163 & 195 & 0.023 \\
\hline Presence of DARA & -0.265 & 197 & $<0.001$ \\
\hline Surface of DARA & -0.343 & 197 & $<0.001$ \\
\hline Location of DARA & 0.400 & 197 & $<0.001$ \\
\hline DARA involving the fovea & -0.386 & 197 & $<0.001$ \\
\hline $\begin{array}{l}\text { DARA within I DD of the } \\
\text { fovea, but sparing the fovea }\end{array}$ & 0.128 & 197 & 0.072 \\
\hline $\begin{array}{l}\text { DARA more than I DD } \\
\text { outside the fovea }\end{array}$ & 0.309 & 197 & $<0.001$ \\
\hline Mean number of recurrences & -0.063 & 176 & 0.409 \\
\hline $\begin{array}{l}\text { Notes: } r \text {, correlation coefficient. Bold } f \\
\text { significant. } \\
\text { Abbreviations: BCVA, best-correcte } \\
\text { pigment epithelium (RPE) alterations; } \\
\text { cystoid retinal degeneration. }\end{array}$ & ont indicate & $\begin{array}{l}\text { s data were cons } \\
\text { ity; DARA, diffus }\end{array}$ & $\begin{array}{l}\text { idered statistically } \\
\text { e atrophic retinal } \\
\text { PCRD, posterior }\end{array}$ \\
\hline
\end{tabular}

outcome did not improve significantly after treatment in this cohort. The final visual outcome was strongly associated with baseline visual acuity, surface of DARA, and the presence of DARA within 1 DD of the fovea.

CSC is considered the fourth most common macular disease associated with SRF and/or intraretinal fluid leakage, after age-related macular degeneration, diabetic macular edema, and retinal vascular occlusions. ${ }^{4}$ A prolonged disease course in CSC is known to cause progressive visual decline and can lead to secondary choroidal neovascularization and/ or polypoidal choroidal vasculopathy. ${ }^{17-19}$ Although there is no consensus on the exact definition of chronicity, the most prevalent retinal and choroidal abnormalities characterizing

Table 5 Frequency of all applied treatments in eyes with severe chronic central serous chorioretinopathy

\begin{tabular}{ll}
\hline Treatment modality & $\begin{array}{l}\text { Frequency and } \\
\text { specifications }\end{array}$ \\
\hline Mean number of treatments per case (range) & $2.4(\mathrm{I}-20)$ \\
Number of untreated cases, $\mathrm{n}(\%)$ & $25(14)$ \\
Total number of PDTs, $\mathrm{n}(\%)$ & $246(60)$ \\
Successful ${ }^{\mathrm{a}}$ PDT, $\mathrm{n}(\%)$ & $153(62)$ \\
PDT modality, $\mathrm{n}(\%)$ & $6(2)$ full settings \\
& $160(66)$ half-dose \\
& $39(16)$ half-time \\
& $6(2)$ half-fluency \\
PDT spot size in $\mu \mathrm{m}$ (range) & $35(14)$ unknown \\
Total number of SMLs, $\mathrm{n}(\%)$ & $4,457(\mathrm{I}, 000-8,800)^{\mathrm{b}}$ \\
Successful ${ }^{\mathrm{a}}$ SMLs, $\mathrm{n}(\%)$ & $8 \mathrm{I}(20)$ \\
Total number of conventional thermal laser & $7(9)$ \\
treatments & $84(20)$ \\
Successful ${ }^{\mathrm{a}}$ conventional thermal laser & \\
treatments, $\mathrm{n}(\%)$ & $54(64)$ \\
\hline
\end{tabular}

Notes: a Successful treatment was defined by a complete resolution of subretinal fluid and/or disappearance of posterior cystoid retinal degeneration. ${ }^{b} \mathrm{An}$ average size is reported in case multiple PDT spots were used.

Abbreviations: PDT, photodynamic therapy; SML, subthreshold micropulse diode laser. 
a chronic course are generally agreed upon. ${ }^{10}$ These general clinical characteristics of chronicity include $\geq 1$ significant multifocal areas of atrophic RPE alterations and/or multifocal areas of leakage. Nevertheless, various clinical presentations exist within cCSC, ranging from mild to severe manifestations with clinical characteristics as described here. Therefore, the diagnosis and treatment decision making may form a challenging task in daily clinical practice when confronted with the clinical spectrum of CSC.

In the present study, only $14 \%$ of the severe cCSC patients had a history of documented acute CSC. Otsuka et al ${ }^{14}$ reported a larger incidence of previous acute episodes $(36 \%$ in 25 severe cCSC cases) as compared to the present study, whereas Yannuzzi et $\mathrm{al}^{20}$ reported that merely $8 \%$ of $25 \mathrm{cCSC}$ patients in their study had previously experienced an acute CSC episode. Therefore, these observations at least indicate that a well observed, clinical episode of acute CSC is not required to evolve to chronicity in CSC and to develop extensive, severe cCSC. Since $73 \%$ of the studied eyes showed signs of chronicity at first presentation, it is conceivable that chronic choroidal leakage and congestion may cause gradual, subclinical damage to the overlying RPE. This may explain why most severe cCSC cases in our cohort showed preexisting signs of long-standing disease at first presentation. We also observed that the area of choroidal hyperpermeability on ICGA was larger than the area of leakage on FA in most cases. This can also be an indication of a more widespread underlying choroidal disease process that is slumbering and gradually damaging the RPE. This process may have started subclinically and without patients notification. Only when there is a RPE outer blood-retinal barrier breakdown with SRF accumulation the vision loss may occur. The multifocal and/or diffuse leakage on FA in some severe cCSC cases can be considered as the "tip of the iceberg" of a larger underlying area of dysfunctional leaking choroid. Eventually, focal and/or diffuse damage to the RPE and the outer blood-retinal barrier can result in more pronounced damage to the overlying neuroretina, for instance, through the development of RPE atrophy, SRF accumulation, and PCRD.

In the present study, final visual outcome was most strongly correlated with baseline BCVA (before severe presentation), location of DARA within 1 DD from the fovea, and DARA surface size. Despite a high percentage of post-treatment SRF resolution in most cases (76\%), final visual outcome did not improve significantly in our cohort. The BCVA at final visit was on average 70 ETDRS letters. In another smaller study, including patients with presumably severe disease, a final BCVA of $>0.5$ Snellen (70 ETDRS letters) was reported in $80 \%$ of the eyes (37/46). ${ }^{14}$ Wang et $\mathrm{al}^{21}$ have postulated that SRF leakage lasting $>4$ months may cause retinal atrophy and irreversible visual loss, but little is known about the exact time that has to ensue before chronic SRF accumulation causes photoreceptor degeneration, neuroretinal atrophy, and consequent vision loss. In the present study, most cases had a disease duration that was far longer than 4 months and experienced multiple recurrences of SRF accumulation. It is therefore plausible to assume that irreversible macular photoreceptor dysfunction due to prolonged disease has contributed to irreversible BCVA loss.

In this study, DARA was the most prevalent finding among severely affected patients, and DARA surface also tended to expand over time. The development of diffuse RPE atrophy has been related to corticosteroid use. ${ }^{22}$ In the present cohort, $18 \%$ of the patients reported corticosteroid use. However, we did not observe a significant negative correlation between corticosteroid use and a poor post-treatment BCVA, confirming our earlier findings in patients with steroid-associated cCSC who were treated with PDT. ${ }^{23}$

Among our studied cases, the majority (87\%) was treated during follow-up. Nevertheless, SRF persisted in $24 \%$ of cases, and PCRD remained in $18 \%$ until final visit. Silva et $\mathrm{al}^{24}$ previously reported a $93 \%$ SRF resolution and a 100\% posttreatment PCRD resolution in a cohort of 46 general cCSC cases. It has been previously suggested that outer retinal ischemia due to chronic retinal detachment may contribute to PCRD development. ${ }^{25,26}$ Despite increasing evidence on the superiority of PDT as first-choice treatment for CSC, a large variability in CSC treatment exists in clinical practice. ${ }^{27,28}$ In this study, PDT with reduced settings was the most commonly used treatment modality, with the highest success rate $(62 \%)$ in resolution of SRF and PCRD. This success rate is lower when compared to literature on outcome of PDT in a general cCSC population, ${ }^{29-31}$ which may be related to the disease severity as well as our strict definition of success: a complete resolution of SRF and PCRD.

In the present study, we proposed a definition for severity in cCSC. The choice of the suggested criteria such as a large cumulative surface of DARA in the macula of $>5$ DD was somewhat arbitrary, based on clinical experience of the involved retina specialists. Future research must establish the validity of these criteria of severity. In order to report a history of acute CSC in this retrospective study, we were dependent on symptomatic disease episodes. Therefore, the abovementioned percentage of patients with a history of acute CSC may be an underestimation, since a small proportion of acute CSC episodes may occur asymptomatically. 
Here, we could demonstrate that patient characteristics including baseline BCVA, total area of DARA, location of DARA, and presence of PCRD can predict up to $47 \%$ of the variance in final BCVA outcome in severe cCSCs. Future prospective studies may provide additional information on the retinal and choroidal vasculature conditions and reveal other prognostic factors associated with progression and visual outcome in cCSC.

\section{Conclusion}

Based on the suggested definition in this study, CSC appears to encompass a clinical spectrum that includes a group of severe cases of cCSC which often respond to treatment, but with a more guarded visual prognosis as compared to cCSC cases without characteristics of severity. Most of these severe cCSC cases already have a chronic phenotype at presentation, whereas only a minority of them have a documented history of acute CSC. This may indicate that acute CSC and (severe) cCSC are relatively distinct phenotypes both clinically and pathophysiologically. Future studies can be directed toward a further distinction of different phenotypes within the CSC spectrum, to gain further insight into possible differences in genetic background, pathophysiology, optimal treatments, and treatment outcome.

\section{Acknowledgments}

This research was supported by the following funding sources: Stichting Leids Oogheelkundig Ondersteuningsfonds, Rotterdamse Stichting Blindenbelangen, Stichting Wetenschappelijk Onderzoek Het Oogziekenhuis, Macula Fonds, Landelijke Stichting voor Blinden en Slechtzienden, Retina the Netherlands, and BlindenPenning, which contributed through UitZicht. Prof Dr Camiel JF Boon was supported by a Gisela Thier Fellowship of Leiden University and a VENI grant of the Netherlands Organization for Scientific Research (NWO). The sponsor or funding organization had no role in the design or conduct of this research.

\section{Disclosure}

The authors report no conflicts of interest in this work.

\section{References}

1. Gass JD. Pathogenesis of disciform detachment of the neuroepithelium. Am J Ophthalmol. 1967;63(3):1-139.

2. Warrow DJ, Hoang QV, Freund KB. Pachychoroid pigment epitheliopathy. Retina. 2013;33(8):1659-1672.

3. Gemenetzi M, De Salvo G, Lotery AJ. Central serous chorioretinopathy: an update on pathogenesis and treatment. Eye. 2010;24(12):1743-1756.

4. Wang M, Munch IC, Hasler PW, Prünte C, Larsen M. Central serous chorioretinopathy. Acta Ophthalmol. 2008;86(2):126-145.
5. Liew G, Quin G, Gillies M, Fraser-Bell S. Central serous chorioretinopathy: a review of epidemiology and pathophysiology. Clin Exp Ophthalmol. 2013;41(2):201-214.

6. Haimovici R, Koh S, Gagnon DR, Lehrfeld T, Wellik S; Central Serous Chorioretinopathy Case-Control Study Group. Risk factors for central serous chorioretinopathy: a case-control study. Ophthalmology. 2004; 111(2):244-249

7. Miki A, Kondo N, Yanagisawa S, Bessho H, Honda S, Negi A. Common variants in the complement factor $\mathrm{H}$ gene confer genetic susceptibility to central serous chorioretinopathy. Ophthalmology. 2014; 121(5):1067-1072.

8. de Jong EK, Breukink MB, Schellevis RL, et al. Chronic central serous chorioretinopathy is associated with genetic variants implicated in agerelated macular degeneration. Ophthalmology. 2015;122(3):562-570.

9. Breukink MB, Schellevis RL, Boon CJ, et al. Genomic copy number variations of the complement component $\mathrm{C} 4 \mathrm{~B}$ gene are associated with chronic central serous chorioretinopathy. Invest Ophthalmol Vis Sci. 2015;56(9):5608-5613.

10. Daruich A, Matet A, Dirani A, et al. Central serous chorioretinopathy: recent findings and new physiopathology hypothesis. Prog Retin Eye Res. 2015;48:82-118.

11. Breukink MB, Dingemans AJ, den Hollander AI, et al. Chronic central serous chorioretinopathy: long-term follow-up and vision-related quality of life. Clin Ophthalmol. 2017;11:39-46.

12. Piccolino FC, De La Longrais RR, Manea M, Cicinelli S. Posterior cystoid retinal degeneration in central serous chorioretinopathy. Retina. 2008;28(7):1008-1012.

13. Balaratnasingam C, Freund KB, Tan AM, et al. Bullous variant of central serous chorioretinopathy: expansion of phenotypic features using multimethod imaging. Ophthalmology. 2016;123(7):1541-1552.

14. Otsuka S, Ohba N, Nakao K. A long-term follow-up study of severe variant of central serous chorioretinopathy. Retina. 2002;22(1):25-32.

15. von Winning $\mathrm{CH}$, Oosterhuis JA, Renger-van Dijk AH, HornstraLimburg H, Polak BC. Diffuse retinal pigment epitheliopathy. Ophthalmologica. 1982;185(1):7-14.

16. Gregori NZ, Feuer W, Rosenfeld PJ. Novel method for analyzing snellen visual acuity measurements. Retina. 2010;30(7):1046-1050.

17. Peiretti E, Ferrara DC, Caminiti G, Mura M, Hughes J. Choroidal neovascularization in Caucasian patients with longstanding central serous chorioretinopathy. Retina. 2015;35:1360-1367.

18. Castro-Correia J, Coutinho MF, Rosas V, Maia J. Long-term follow-up of central serous retinopathy in 150 patients. Doc Ophthalmol. 1992; 81(4):379-386.

19. Park HS, Kim IT. Clinical characteristics of polypoidal choroidal vasculopathy associated with chronic central serous chorioretionopathy. Korean J Ophthalmol. 2012;26:15-20.

20. Yannuzzi LA, Shakin JL, Fisher YL, Altomonte MA. Peripheral retinal detachments and retinal pigment epithelial atrophic tracts secondary to central serous pigment epitheliopathy. 1984. Retina. 2012; 32(suppl 1):1554-1572.

21. Wang MS, Sander B, Larsen M. Retinal atrophy in idiopathic central serous chorioretinopathy. Am J Ophthalmol. 2002;133:787-793.

22. Polak BC, Baarsma GS, Snyers B. Diffuse retinal pigment epitheliopathy complicating systemic corticosteroid treatment. Br J Ophthalmol. 1995;79(10):922-925.

23. Breukink MB, Mohabati D, van Dijk EH, et al. Efficacy of photodynamic therapy in steroid-associated chronic central serous chorioretinopathy: a case-control study. Acta Ophthalmol. 2016;94(6):565-572.

24. Silva RM, Ruiz-Moreno JM, Gomez-Ulla F, et al. Photodynamic therapy for chronic central serous chorioretinopathy: a 4-year follow-up study. Retina. 2013;33(2):309-315.

25. Iida T, Yannuzzi LA, Spaide RF, Borodoker N, Carvalho CA, Negrao S. Cystoid macular degeneration in chronic central serous chorioretinopathy. Retina. 2003;23(1):1-7.

26. Gaudric A. Macular cysts, holes and cavitations: 2006 Jules Gonin lecture of the Retina Research Foundation. Graefes Arch Clin Exp Ophthalmol. 2008;246(7):1071-1079. 
27. Salehi M, Wenick AS, Law HA, Evans JR, Gehlbach P. Interventions for central serous chorioretinopathy: a network meta-analysis. Cochrane Database Syst Rev. 2015;22(12):Cd011841.

28. Mehta PH, Meyerle C, Sivaprasad S, Boon C, Chhablani J. Preferred practice pattern in central serous chorioretinopathy. Br J Ophthalmol. 2017;101(5):587-590.

29. Nicolo M, Eandi CM, Alovisi C, et al. Half-fluence versus half-dose photodynamic therapy in chronic central serous chorioretinopathy. Am J Ophthalmol. 2014;157(5):1033-1037.
30. Lim JI, Glassman AR, Aiello LP, et al. Collaborative retrospective macula society study of photodynamic therapy for chronic central serous chorioretinopathy. Ophthalmology. 2014;121(5):1073-1078.

31. Lai FH, Ng DS, Bakthavatsalam M, et al. A multicenter study on the long-term outcomes of half-dose photodynamic therapy in chronic central serous chorioretinopathy. Am J Ophthalmol. 2016;170:91-99.

\section{Publish your work in this journal}

Clinical Ophthalmology is an international, peer-reviewed journal covering all subspecialties within ophthalmology. Key topics include: Optometry; Visual science; Pharmacology and drug therapy in eye diseases; Basic Sciences; Primary and Secondary eye care; Patient Safety and Quality of Care Improvements. This journal is indexed on

\section{Dovepress}

PubMed Central and CAS, and is the official journal of The Society of Clinical Ophthalmology (SCO). The manuscript management system is completely online and includes a very quick and fair peer-review system, which is all easy to use. Visit http://www.dovepress.com/ testimonials.php to read real quotes from published authors. 